Article

\title{
The PoGO+ Balloon-Borne Hard X-ray Polarimetry Mission
}

\author{
Mette Friis ${ }^{1,2}$, Mózsi Kiss ${ }^{1,2, *(1)}$, Victor Mikhalev ${ }^{1,2}$ (D), Mark Pearce ${ }^{1,2}$ (D) and \\ Hiromitsu Takahashi ${ }^{3,+}$ \\ 1 KTH Royal Institute of Technology, Department of Physics, 10691 Stockholm, Sweden; mettef@kth.se (M.F.); \\ mikhalev@kth.se (V.M.); pearce@kth.se (M.P.) \\ 2 The Oskar Klein Centre for Cosmoparticle Physics, AlbaNova University Center, 10691 Stockholm, Sweden \\ 3 Department of Physical Science, Hiroshima University, Hiroshima 739-8526, Japan; \\ hirotaka@astro.hiroshima-u.ac.jp \\ * Correspondence: mozsi@kth.se; Tel.: +46-73-765-24-50 \\ + On behalf of the PoGO+ Collaboration.
}

Received: 31 January 2018; Accepted: 26 February 2018; Published: 2 March 2018

\begin{abstract}
The PoGO mission, including the PoGOLite Pathfinder and PoGO+, aims to provide polarimetric measurements of the Crab system and Cygnus X-1 in the hard X-ray band. Measurements are conducted from a stabilized balloon-borne platform, launched on a 1 million cubic meter balloon from the Esrange Space Center in Sweden to an altitude of approximately $40 \mathrm{~km}$. Several flights have been conducted, resulting in two independent measurements of the Crab polarization and one of Cygnus X-1. Here, a review of the PoGO mission is presented, including a description of the payload and the flight campaigns, and a discussion of some of the scientific results obtained to date.
\end{abstract}

Keywords: Compton polarimeter; hard X-rays; Crab, Cygnus X-1; scientific ballooning; payload design, attitude control

\section{Introduction}

PoGO+ is a balloon-borne hard X-ray Compton polarimetry telescope operating in the energy range $20-180 \mathrm{keV}$. "PoGO" stands for "Polarized Gamma-ray Observer," and the plus signifies an upgrade [1], specifically from the PoGOLite "Pathfinder" [2], which was launched in 2013 [3]. The instrument is optimized for point-sources and has a field of view collimated to about $2^{\circ}$. Observational targets are the X-ray-bright sources on the northern hemisphere: the Crab and Cygnus X-1.

By providing two new observational parameters, namely the polarization fraction and polarization angle, polarimetry is expected to provide new insight into the emission mechanisms, magnetic fields, and geometries of sources observed. The relevance of polarimetric studies has been extensively discussed [4], and results have been reported in particular for the Crab system. Many of the results suffer, however, from systematic effects, leaving open questions about the emission mechanisms at work in the observed astrophysical sources. The goal of the PoGO mission is to provide statistically constrained polarimetric observations (a minimum detectable polarization of $\sim 10 \%$ before background subtraction), in a previously unexplored energy range, with well-understood systematic effects, using an instrument that has been extensively calibrated with both polarized and unpolarized beams [5].

Here, developments of the PoGO mission are collected, leading up to the 2016 flight and a summary of observational results for the Crab and Cygnus X-1. Section 2 gives a description of the PoGO+ payload, including the instrument, attitude control system, and gondola structure. A brief review of the flight campaigns to date, and the lessons learned from these, is provided in Section 3. 
In Section 4, scientific results are presented. Finally, Section 5 provides an outlook on some of the studies that are currently on-going using data collected with PoGO+.

\section{The PoGO+ Payload}

PoGO+ uses a 61-pixel array of plastic scintillators for reconstructing azimuthal scattering angles from two-unit interactions (polarization candidate events) in temporal coincidence. As per the Klein-Nishina formula, such scattering angles will be modulated by the polarization of the incident photon beam, following a sinusoidal distribution with a $180^{\circ}$ period [6]. The scintillators, type EJ-204 from Eljen Technology, are $12 \mathrm{~cm}$ tall and about $3 \mathrm{~cm}$ across, with a hexagonal cross section, allowing them to be tightly packed in a honeycomb structure. Copper tubes, approximately $70 \mathrm{~cm}$ in length, are used to collimate the field of view. These are made from $0.5 \mathrm{~mm}$ thin copper sheets, folded into hexagonal cross-section tubes, which are wrapped in $200 \mu \mathrm{m}$ of tin foils followed by the same thickness of lead foils. This "graded shield" construction of the collimators efficiently suppresses the out-of-aperture background of the measurements. The plastic scintillators are surrounded on the bottom and on the sides by a bismuth germanium oxide (BGO) anticoincidence shield, segmented into 61 pieces on the bottom of the instrument (one corresponding to each plastic scintillator) and 30 pieces on the side. The anticoincidence shield is $60 \mathrm{~cm}$ tall (compared to the $12 \mathrm{~cm}$ height of the plastic scintillators) and the thickness exceeds $3 \mathrm{~cm}$ on each side. Plastic scintillators and side anticoincidence shield elements are read out by the same type of photo-multiplier tube, a modified version of Hamamatsu R7899. In order to cancel out variations in response between individual detector elements, the entire detector array rotates during observations at a rate of $1^{\circ} / \mathrm{s}$. The dominant background is from atmospheric neutron interactions [7], wherefore the entire detector array is surrounded by a passive polyethylene shield, with a thickness between 5 and $15 \mathrm{~cm}$. Figure 1 illustrates the detector design. A more comprehensive description of the payload is presented in [2].

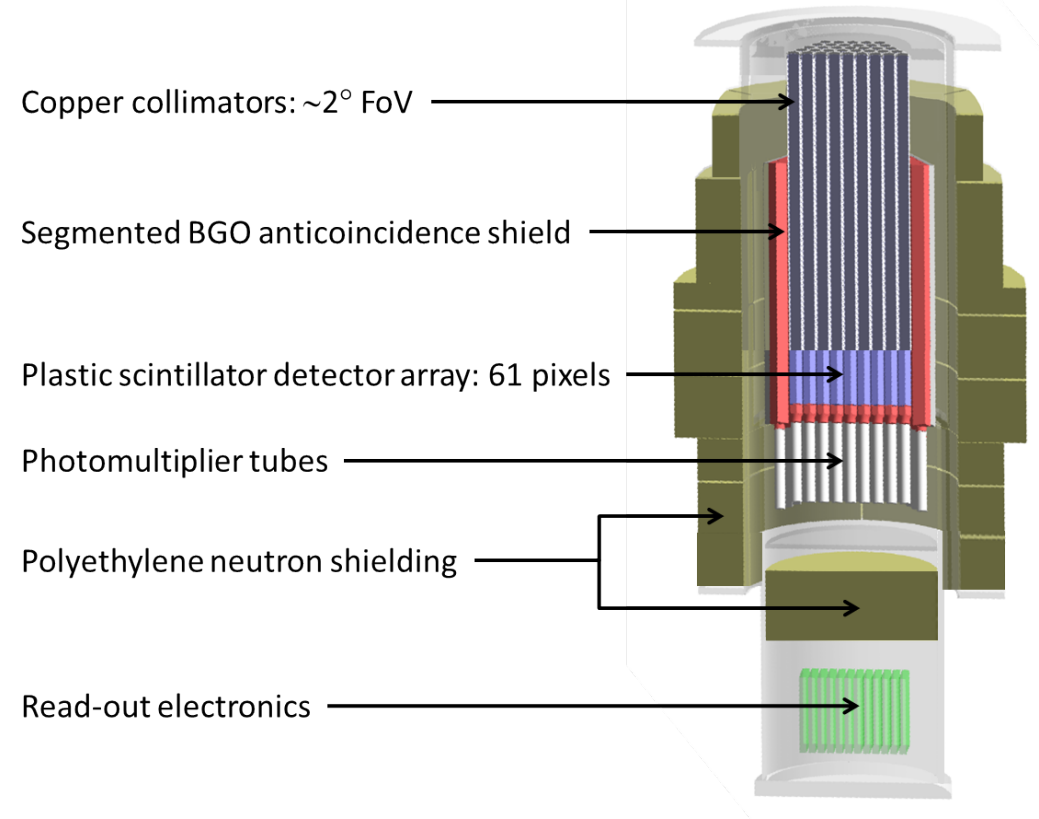

Figure 1. Sketch of the PoGO+ polarimeter. The height is $\sim 1.5 \mathrm{~m}$, and the total weight is $\sim 600 \mathrm{~kg}$.

Celestial targets are acquired and tracked using a custom attitude control system and gimbal unit assembly, designed and developed by DST Control, Sweden. In order to prevent degradation of the polarimetric response due to shadowing effects from the collimators, the pointing is required to be better than $5 \%$ of the instrument field of view, i.e., within $\sim 0.1^{\circ}$. Coarse azimuthal pointing is achieved using a flight train motor coupled directly to the interface to the balloon rigging. A flywheel provides 
fine-tuning in azimuth, and excess momentum can be transferred to the balloon through a momentum dump system that controls the feedback to the flight train. Brushless direct drive torque motors are used throughout, with a configuration providing excellent heat dissipation and electromagnetic compatibility. A detailed description of the attitude control system hardware and design philosophy is presented in [8]. The gimbal unit assembly is shown in Figure 2.

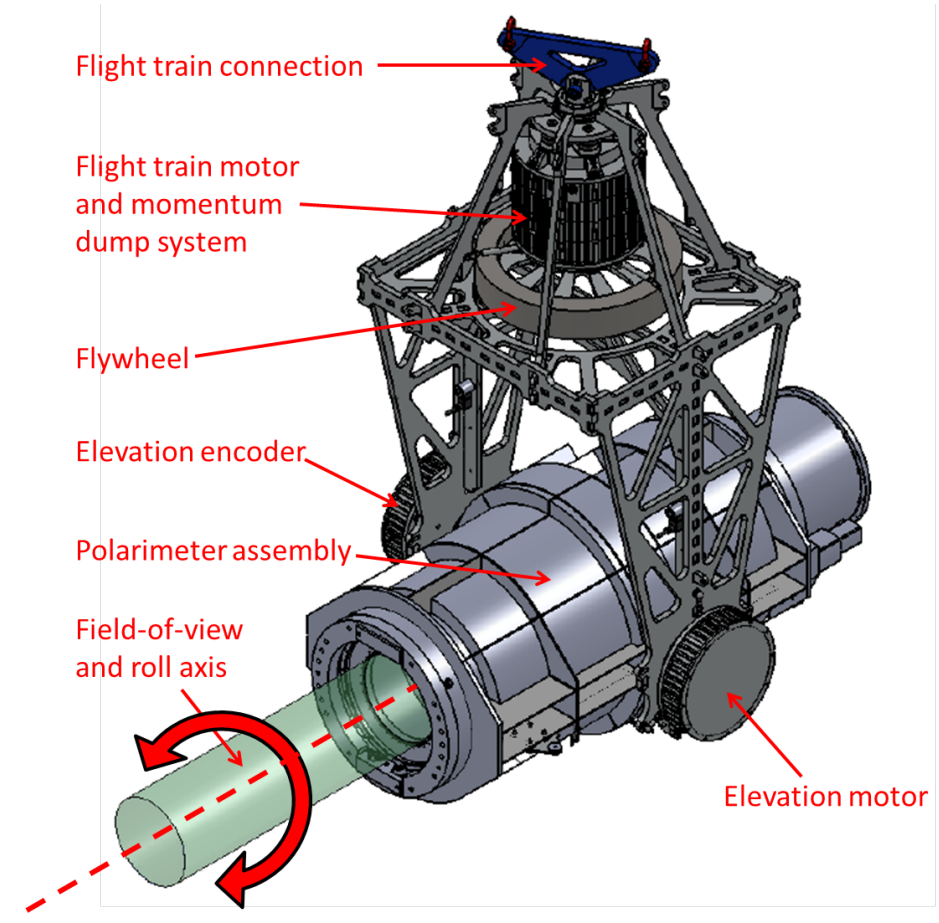

Figure 2. Sketch of the gimbal unit assembly housing the polarimeter. The framework stands $\sim 2 \mathrm{~m}$ tall and has a mass of $\sim 300 \mathrm{~kg}$, excluding the polarimeter.

Pointing solutions and motor control feedbacks are calculated based on several attitude inputs. An encoder mounted on the polarimeter axle provides the telescope elevation. For the azimuth, the backbone of the system is a differential GPS which, utilizing a $10 \mathrm{~m}$ baseline, can provide the desired pointing accuracy in itself. A three-axis magnetometer serves as a backup in case of a GPS failure. In the upgrade from PoGOLite to PoGO+, a sun tracker was additionally included. Using a two-dimensional position-sensitive device inside a free-moving gimbal, which automatically locates and follows the sun, this tracker can provide the instrument's azimuth based on its location and the position of the sun. These sensors provide absolute information for instrument pointing. Relative feedback is provided by an optical star tracker camera, mounted co-axially with the polarimeter. For each X-ray target, an optical guide star is defined. The camera identifies the pixel coordinates of the guide star and matches these with the expected coordinates, calculated in real time based on the instrument position, altitude, and time of day, correcting for the field rotation of the star field as time goes by. In this tracking mode, motor feedbacks are generated based on the difference between the expected and the observed pixel coordinates of the relevant star. An inclinometer mounted on the gimbal unit assembly provides information on the gondola pitch and roll. In addition, a micro-mechanical inertial measurement unit is used, providing an additional layer of redundancy. The unit is continuously augmented by inputs from the other available sensors and can, in case other attitude sensors fail, retain attitude information for some time, until the remaining systems become operational again.

The polarimeter and gimbal unit assembly are housed inside a gondola structure designed and manufactured by SSC Esrange. The structure provides thermal shielding, mechanical stiffness for pointing stability, and protection during launch and landing. It consists of a six-sided light-weight 
framework with aluminum ribs and honeycomb composite plates. Solar panels and booms for the GPS and communication antennas are also affixed onto the structure, as is a radiator for expelling heat from the polarimeter electronics, using a fluid-based cooling system. During assembly and disassembly, the gondola can be separated into an upper part, housing the polarimeter, and a lower part, which contains batteries, communications equipment, and ancillary hardware. The assembled PoGO+ payload is shown in Figure 3.

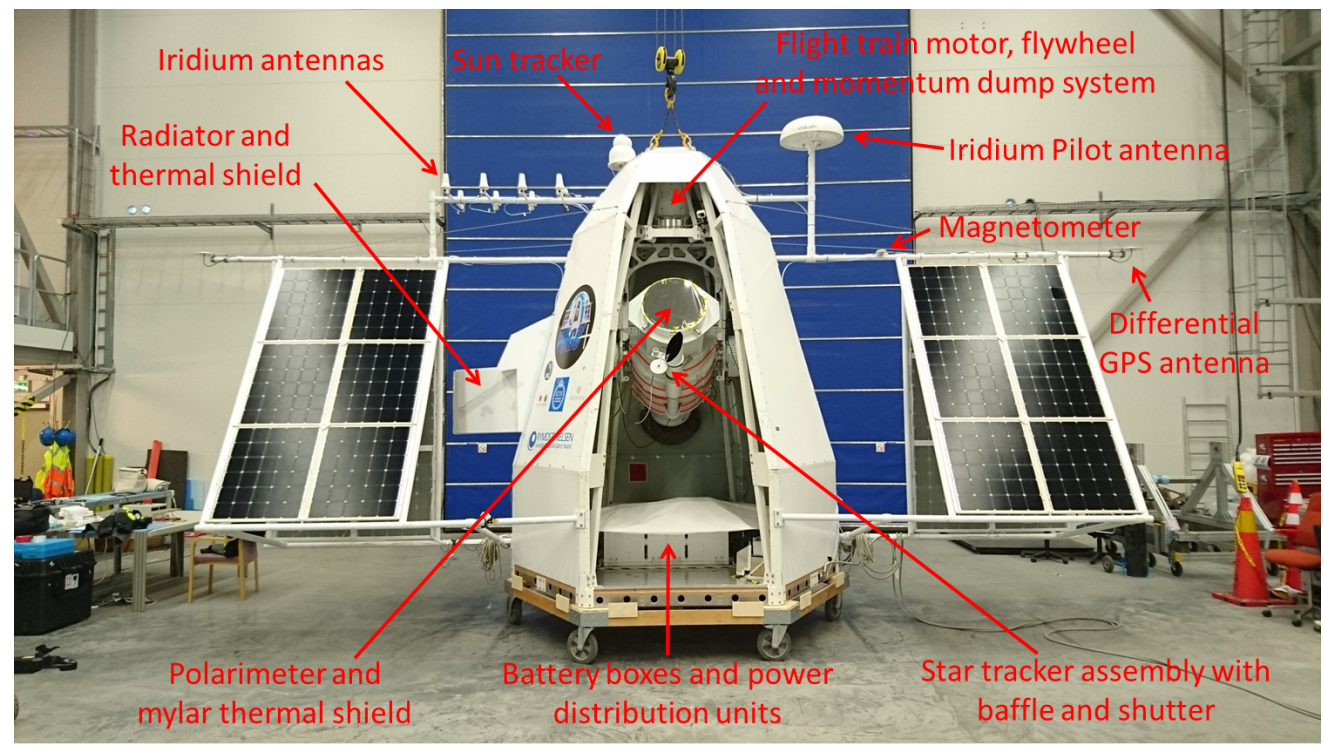

Figure 3. The PoGO+ payload and its various constituents. Crash pads, ballast hoppers, and line-of-sight antennas mounted underneath the gondola are not shown. One panel of the gondola, the front where the polarimeter is looking out, has been removed, showing components inside. The structure is $\sim 4 \mathrm{~m}$ tall and the widest part, at the GPS booms, is $\sim 10 \mathrm{~m}$. For flight, the total suspended weight is almost two tonnes, which includes $450 \mathrm{~kg}$ of ballast.

\section{PoGO Flight Campaigns}

PoGO campaigns are conducted from the Swedish Space Corporation (SSC) Esrange Space Center in Northern Sweden $\left(67.89^{\circ} \mathrm{N}, 21.08^{\circ} \mathrm{E}\right)$. During the summer, circumpolar winds at a $\sim 40 \mathrm{~km}$ altitude move in the westerly direction, allowing flights from Esrange to Canada. The launch window opens around 1 July, when the angular separation between the Crab and the sun exceeds $15^{\circ}$, and closes during August, when the circumpolar vortex starts disintegrating. There have been four campaigns to date, resulting in three launches.

The year 2011 saw the first test launch of the PoGOLite "Pathfinder'.' A leak of helium from the balloon forced the flight to be terminated prematurely, resulting in a landing near Nikkaluokta, approximately $100 \mathrm{~km}$ to the west of the Esrange Space Center. Float altitude was never achieved, so scientific measurements could not be conducted. At landing, only minor damage was incurred, and the polarimeter array could easily be repaired from spare detector units.

A second launch attempt was foreseen for the summer of 2012. Due to unfavorable weather conditions, the payload remained grounded for the duration of the launch window.

A successful launch of the PoGOLite "Pathfinder" took place on 12 July 2013. For this particular launch, an overflight agreement had also been secured from Russian authorities, opening up the possibility for a full polar circumnavigation, concluding with a termination somewhere over Scandinavia. In the end, due to a predicted drift in the trajectory towards higher latitudes, whereby landing in Scandinavia would no longer be certain, the flight was terminated over Russia on 25 July, resulting in a landing near Norilsk, $\sim 3000 \mathrm{~km}$ east of Moscow. Details of this flight are presented in [2]. Due to issues related to overheating, scientific operations could only be concluded during the first 
three days of the flight. A statistics-limited measurement of the Crab polarization could, however, be reported [3].

The next launch took place on 12 July 2016, now with the upgraded payload, called "PoGO+". PoGO+ benefited tremendously from data collected during the 2013 flight of PoGOLite. The polarimeter was extensively redesigned and optimized [1], including (i) new detector units and improved reflective wrapping yielding higher sensitivity; (ii) improved front-end electronics with a wider dynamic range and a higher waveform sampling rate providing improved efficiency for the pulse shape discrimination and event selection; (iii) additional polyethylene neutron shielding for suppressing the flight background; and (iv) higher sensitivity for the side anticoincidence shield, yielding a lower energy threshold for the background rejection. On the gondola side, an Iridium Pilot antenna had been added, allowing for better monitoring, operations, and data download rates during over-the-horizon communications. For the attitude information, the aforementioned sun tracker had been added, which simplified observations of the Crab system, where close angular proximity to the sun had previously complicated automatic pointing operations based on feedback from the optical star tracker camera. Of particular importance for the scientific observations was the decision to intersperse background measurements with the on-source observations of the Crab and Cygnus X-1. Throughout the flight, background fields separated by $5^{\circ}$ to the east and west of the target were observed, with transitions to/from the X-ray source occurring automatically approximately every $15 \mathrm{~min}$, and with almost equal time spent on source and on background fields. This strategy allowed the temporal evolution of the background rate and its residual polarization to be studied, and was a prerequisite for correctly determining the in-flight signal-to-background rate, needed for the background-subtraction of the flight data. The omission of such interspersed background would have resulted in a significant degradation of the systematic precision of the polarimetric results. Figure 4 shows an example of signal (on-source) and background (off-source) count rates during a Crab observation, underlining the importance of interspersing the two kinds of observations in this fashion.

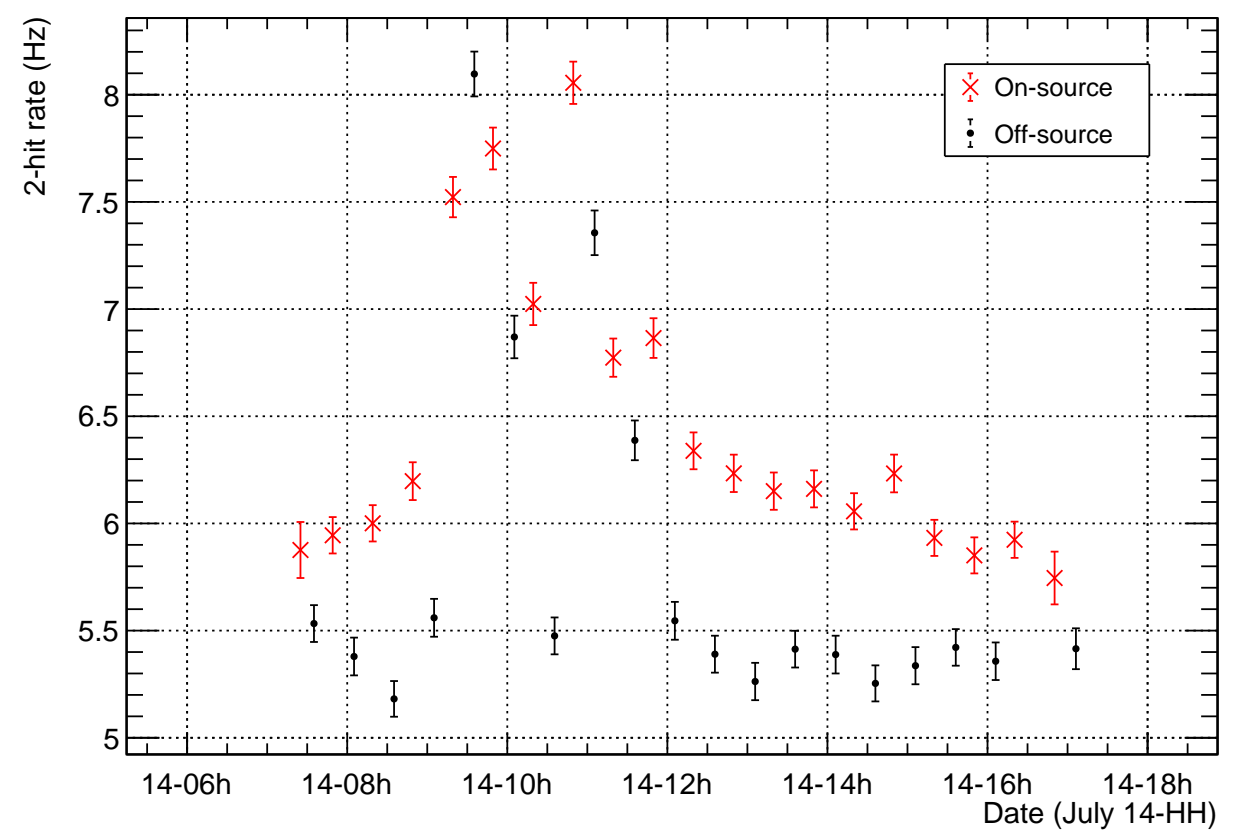

Figure 4. Count rate of two-hit events during the Crab observation on 14 July 2016. Apart from the smooth change as a function of time resulting from the sidereal motion of the source across the sky, large fluctuations can be seen both in the on-source (red) and off-source (black) data points. In the absence of interspersed background measurements, this would be indistinguishable from a change in the source rate only, resulting in an incorrectly determined signal-to-background ratio. 
Polarimetric results are derived from the azimuthal scattering angle distributions of these two-hit events. Recorded scattering angles are corrected for the rotation of the detector array throughout the measurement, as well as for the instantaneous gondola roll, sampled at $4 \mathrm{~Hz}$. While distributions for both on-source and off-source measurements are first inspected in terms of $\chi^{2}$ per degrees of freedom goodness-of-fit with respect to $180^{\circ}$ (polarization) and $360^{\circ}$ (anisotropic background) components, an unbinned Stokes-based analysis is used to determine final results. Background is subtracted using the signal-to-background ratio, following corrections for the slight change in acquisition live-time when observing on-source versus off-source. Flight results are summarized in the next section.

\section{Scientific Results from PoGO}

Polarimetric results reported for the high-energy emission of the Crab are limited to a few data points only. At low energies, instruments on the OSO-8 satellite measured polarization at 2.6 and $5.2 \mathrm{keV}$ already in the 1970s [9]. For the higher energies of several hundred keV, results are reported from the INTEGRAL SPI [10,11], and INTEGRAL IBIS [12,13] instruments. The reliability of the INTEGRAL results may, however, be limited by the fact that these instruments were not designed as polarimeters and were not calibrated for polarimetry in ground testing campaigns. PoGOLite, through the "Pathfinder" mission, provided the first polarimetric results in the intermediate energy range, 20-120 keV [3]. The upgraded PoGO+ instrument later provided an independent study in the range 19-160 keV, confirming the findings from PoGOLite, significantly improving the statistical precision and producing a result for the off-pulse (nebula-dominated) and second pulse ("P2", off-pulse-subtracted) phase regions, respectively [14]. Since the PoGO+ results were published, results from the AstroSat mission have also been reported [15], in an energy range complementary to that of PoGO+, 100-380 keV. Results from these high-energy studies were collected and are shown in Figure 5, where low-energy optical values from the NOT [16] are also included.
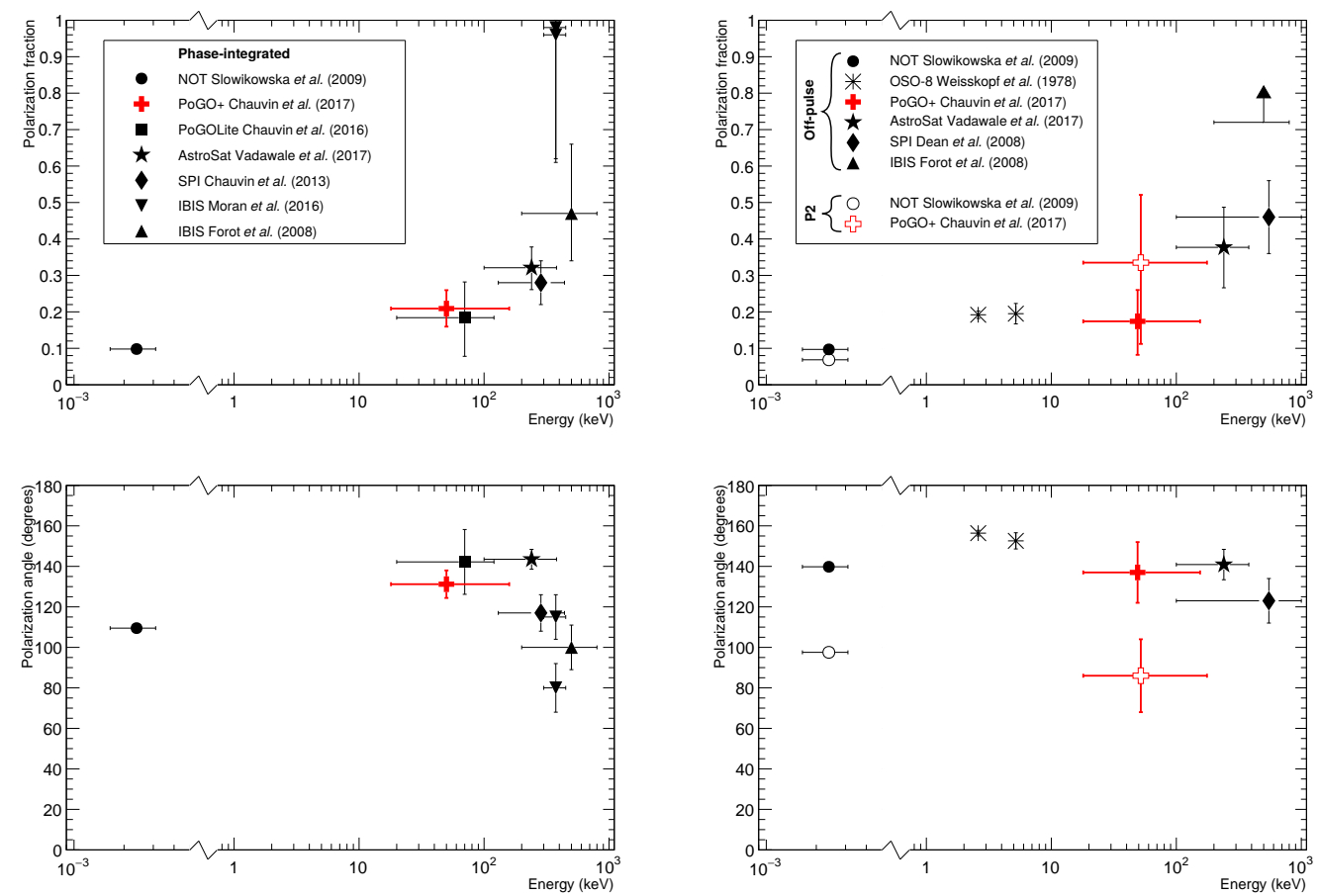

Figure 5. Results from PoGOLite and PoGO+ with other polarimetric studies in different high-energy emission regimes, for the phase-integrated Crab measurement (left); and the off-pulse (nebula-dominated) and P2 (off-pulse-subtracted) phase intervals (right), for the polarization fraction (upper panels) and polarization angle (lower panels). Low-energy optical results are also shown. 
The PoGO+ results [14] reveal a polarization fraction of $(20.9 \pm 5.0) \%$ for the phase-integrated $\mathrm{Crab}$ measurement. This relatively high value indicates synchrotron radiation from a magnetically ordered and therefore compact emission region. The observed polarization angle is $(131.3 \pm 6.8)^{\circ}$, compatible with the pulsar spin axis which is identified as having angle $(124.0 \pm 0.1)^{\circ}$ [17] projected on the sky. NuSTAR imaging [18] shows that high energy emission is predominantly coming from more central regions of the Crab system. The alignment of the measured polarization angle with the pulsar spin axis is thus consistent with the toroidal ring region [19] of the Crab system dominating the hard X-ray emission observed by PoGO+. Spatially resolved measurements from HST [20] reveal high levels of polarization from the synchrotron knot and from the wisp structures, exhibiting polarization angles of $(124.7 \pm 1.0)^{\circ}$ and $124^{\circ}-130^{\circ}$, respectively. A vector map of the full nebula instead shows a peak distribution around polarization angle $165^{\circ}$. Such fine details are not resolved in hard X-rays, but the agreement with the polarization angle determined by PoGO+ suggests that these features have counterparts also in the energy range of the latter instrument, $\sim 19-160 \mathrm{keV}$.

Taken at face value, published polarimetric results for the $\mathrm{Crab}$ from different instruments indicate an increase of polarization fraction as a function of energy. However, recent results from AstroSat [15] are in agreement with PoGO results [14], which do not show a drastic increase as seen by INTEGRAL IBIS $[12,13]$. This strain illustrates the difficulty of deriving polarimetric results, polarization fraction in particular, from instruments not calibrated as polarimeters.

\section{Summary and Outlook}

Unlike satellite-based measurements, scientific ballooning missions allow modifications and upgrades to the payload design to be implemented based on flight data and experience, increasing the chance of success in a re-flight. In this fashion, PoGO+ has successfully conducted polarimetric observations of the Crab system and Cygnus X-1 in the energy range $\sim 20-180 \mathrm{keV}$, based on experience from the flight of the PoGOLite "Pathfinder." Results presented for the Crab [14] are the first in this energy range. Independent results from AstroSat [15] have been found to be in agreement, strengthening the confidence in the results and countering a rapid increase of polarization fraction with energy as suggested by some measurements [12,13]. A follow-up to the AstroSat observations, and the claim [15] of varying polarization properties in the Crab off-pulse region, has also been conducted [22], wherein PoGO+ data has been revisited, allowing this claim to be addressed. For Cygnus X-1, PoGO+ results [21] allow the emission geometry predicted by different models to be constrained.

What is currently being investigated is the possibility of an energy-dependent analysis of the polarization data, for the Crab and/or for Cygnus X-1. As PoGO+ is optimized for polarimetry and not calorimetry, this would be a Monte-Carlo-driven study, relying on the previously benchmarked simulation results [5].

There are currently no plans to re-fly PoGO+, but the experience from the PoGO mission is used for the development of a new instrument, called SPHiNX ("Satellite Polarimeter for High eNergy X-rays") [23], which has a wide field of view and is intended for gamma-ray bursts.

Acknowledgments: This research was supported in Sweden by The Swedish National Space Board, The Knut and Alice Wallenberg Foundation, and The Swedish Research Council. In Japan, support was provided by the Japan Society for Promotion of Science and ISAS/JAXA. SSC are thanked for providing expert mission support and launch services at Esrange Space Center. DST Control developed the PoGO+ attitude control system under the leadership of J.-E. Strömberg. Contributions from past collaboration members and students are acknowledged.

Author Contributions: M.F., V.M., M.P. and H.T. attended the Workshop on behalf of the Collaboration. The manuscript was prepared by M.K. All authors have read the manuscript and contributed comments.

Conflicts of Interest: The authors declare no conflict of interest. 


\section{References}

1. Chauvin, M.; Jackson, M.; Kawano, T.; Kiss, M.; Kole, M.; Mikhalev, V.; Moretti, E.; Takahashi, H.; Pearce, M. Optimising a balloon-borne polarimeter in the hard X-ray domain: From the PoGOLite Pathfinder to PoGO+. Astropart. Phys. 2016, 82, 99-107.

2. Chauvin, M.; Florén, H.H.; Jackson, M.; Kamae, T.; Kawano, T.; Kiss, M.; Kole, M.; Mikhalev, V.; Moretti, E.; Olofsson, G.; et al. The design and flight performance of the PoGOLite Pathfinder balloon-borne hard X-ray polarimeter. Exp. Astron. 2016, 41, 17-41.

3. Chauvin, M.; Florén, H.H.; Jackson, M.; Kamae, T.; Kawano, T.; Kiss, M.; Kole, M.; Mikhalev, V.; Moretti, E.; Olofsson, G.; et al. Observation of polarized hard X-ray emission from the Crab by the PoGOLite Pathfinder. Mon. Not. Roy. Astron. Soc. 2016, 456, L84-L88.

4. Marin, F. (Ed.) The Bright Future of Astronomical X-ray Polarimetry; MDPI: Basel, Switzerland, 2018.

5. Chauvin, M.; Friis, M.; Jackson, M.; Kawano, T.; Kiss, M.; Mikhalev, V.; Ohashi, N.; Stana, T.; Takahashi, H.; Pearce, M. Calibration and performance studies of the balloon-borne hard X-ray polarimeter PoGO+. Nucl. Instrum. Meth. A 2017, 859, 125-133.

6. Lei, F.F.; Dean, A.A.; Hills, G.L. Compton Polarimetry in Gamma-Ray Astronomy. Space Sci. Rev. 1997, 82, 309-388.

7. Kole, M.; Pearce, M.; Salinas, M.M. A model of the cosmic ray induced atmospheric neutron environment. Astropart. Phys. 2015, 62, 230-240.

8. Strömberg, J.-E. A Modular Solution for Science Platform Stabilisation. In Proceedings of the 20th Symposium on European Rocket and Balloon Programmes and Related Research; Ouwehand, L., Ed.; ESA Communications ESTEC: Noordwijk, The Netherlands, 2011; pp. 353-358.

9. Weisskopf, M.M.; Silver, E.E.; Kestenbaum, H.H.; Long, K.K.; Novick, R. A precision measurement of the X-ray polarisation of the Crab nebula without pulsar contamination. Astrophys. J. Lett. 1978, 220, L117-L121.

10. Chauvin, M.; Roques, J.J.; Clark, D.D.; Jourdain, E. Polarimetry in the hard X-ray domain with INTEGRAL SPI. Astrophys. J. 2013, 769, 137.

11. Dean, A.A.; Clark, D.D.; Stephen, J.J.; McBride, V.V.; Bassani, L.; Bazzano, A.; Bird, A.A.; Hill, A.A.; Shaw, S.S.; Ubertini, P. Polarized Gamma-Ray Emission from the Crab. Science 2008, 321, 1183-1185.

12. Moran, P.; Kyne, G.; Gouiffès, C.; Laurent, P.; Hallinan, G.; Redfern, R.M.; Shearer, A. A recent change in the optical and $\gamma$-ray polarization of the Crab nebula and pulsar. Mon. Not. Roy. Astron. Soc. 2016, 456, 2974-2981.

13. Forot, M.; Laurent, P.; Grenier, I.I.; Gouiffès, C.; Lebrun, F. Polarization of the Crab pulsar and nebula as observed by the INTEGRAL/IBIS Telescope. Astrophys. J. Lett. 2008, 688, L29-L32.

14. Chauvin, M.; Florén, H.-G.; Friis, M.; Jackson, M.; Kamae, T.; Kataoka, J.; Kawano, T.; Kiss, M.; Mikhalev, V.; Mizuno, T.; et al. Shedding new light on the Crab with polarized X-rays. Sci. Rep. 2017, 7, 7816.

15. Vadawale, S.S.; Chattopadhyay, T.; Mithun, N.N.; Rao, A.A.; Bhattacharya, D.; Vibhute, A.; Bhalerao, V.V.; Dewangan, G.G.; Misra, R.; Paul, B.; et al. Phase-resolved X-ray polarimetry of the Crab pulsar with the AstroSat CZT Imager. Nat. Astron. 2018, 2, 50-55.

16. Słowikowska, A.; Kanbach, G.; Kramer, M.; Stefanescu, A. Optical polarization of the Crab pulsar: Precision measurements and comparison to the radio emission. Mon. Not. Roy. Astron. Soc. 2009, 397, 103-123.

17. Ng, C.-Y.; Romani, R.W. Fitting pulsar wind tori. Astrophys. J. 2004, 601, 479-484.

18. Madsen, K.K.; Reynolds, S.; Harrison, F.; An, H.; Boggs, S.; Christensen, F.F.; Craig, W.W.; Fryer, C.C.; Grefenstette, B.B.; Hailey, C.C.; et al. Broadband X-ray imaging and spectroscopy of the Crab nebula and pulsar with NuSTAR. Astrophys. J. 2015, 801, 66.

19. Nakamura, Y.; Shibata, S. Polarization of the Crab Nebula with disordered magnetic components. Mon. Not. Roy. Astron. Soc. 2007, 381, 1489-1498.

20. Moran, P.; Shearer, A.; Mignani, R.R.; Słowikowska, A.; De Luca, A.; Gouiffès, C.; Laurent, P. Optical polarimetry of the inner Crab nebula and pulsar. Mon. Not. Roy. Astron. Soc. 2013, 433, 2564-2575.

21. Chauvin, M.; Florén, H.-G.; Friis, M.; Jackson, M.; Kamae, T.; Kataoka, J.; Kawano, T.; Kiss, M.; Mikhalev, V.; Mizuno, T.; et al. A constraint on the accretion geometry of the black hole in Cygnus X-1 with X-ray polarimetry. Submitted. 
22. Chauvin, M.; Florén, H.-G.; Friis, M.; Jackson, M.; Kamae, T.; Kataoka, J.; Kawano, T.; Kiss, M.; Mikhalev, V.; Mizuno, T.; et al. The PoGO+ view on Crab off-pulse hard X-ray polarisation. arXiv 2018, arXiv:1802.07775.

23. Xie, F.; Pearce, M. A study of background conditions for SPHiNX-the satellite-borne gamma-ray burst polarimeter. Galaxies 2018, submitted.

(C) 2018 by the authors. Licensee MDPI, Basel, Switzerland. This article is an open access article distributed under the terms and conditions of the Creative Commons Attribution (CC BY) license (http://creativecommons.org/licenses/by/4.0/). 